\title{
A versatile tandem RNA isolation procedure to capture in vivo formed mRNA-protein complexes
}

Ana M. Matia-González ${ }^{1}$, Valentina Iadevaia ${ }^{1}$ \& André P. Gerber*

Dept. of Microbial and Cellular Sciences, School of Biosciences and Medicine, Faculty of

Health and Medical Sciences, University of Surrey, Guildford, GU2 7XH, United Kingdom.

${ }^{1}$ These authors contributed equally to this work.

*Corresponding author:

André P. Gerber

Dept. of Microbial and Cellular Sciences

School of Biosciences and Medicine

Faculty of Health and Medical Sciences

University of Surrey

Stag Hill Campus, 19AX01

Guildford, GU2 7XH

United Kingdom

Tel: $\quad+44(0) 1483682158$

Email: a.gerber@surrey.ac.uk 


\begin{abstract}
We describe a tandem RNA isolation procedure (TRIP) that enables purification of in vivo formed messenger ribonucleoprotein (mRNP) complexes. The procedure relies on the purification of polyadenylated mRNAs with oligo(dT) beads from cellular extracts, followed by the capture of specific mRNAs with 3'-biotinylated 2'-O-methylated antisense RNA oligonucleotides, which are recovered with streptavidin beads. TRIP was applied to isolate in vivo crosslinked mRNP complexes from yeast, nematodes and human cells for subsequent analysis of RNAs and bound proteins. The method provides a basis for adaptation to other types of polyadenylated RNAs, enabling the comprehensive identification of bound proteins/RNAs, and the investigation of dynamic rearrangement of mRNPs imposed by cellular or environmental cues.
\end{abstract}

Keywords: RNA-binding protein; RNA-protein interaction; ribonucleoprotein complex; posttranscriptional regulation; antisense oligonucleotides; non-coding RNA 


\section{Introduction}

Messenger RNAs (mRNAs) are dynamically controlled by hundreds of RNA-binding proteins (RBPs) and non-coding RNAs such as microRNAs (miRNAs) [1, 2]. Thereby, RBPs and associated miRNAs bind to distinct elements in the mRNA, forming so-called ribonucleoprotein (RNP) complexes, which control the localization, translation and decay of mRNAs [3, 4]. Thus, the isolation and molecular characterization of RNPs is fundamental to understand the fate of mRNAs and post-transcriptional gene control.

Several complementary protein and RNA "centric" approaches have been developed to isolate and characterize RNA-protein complexes (reviewed in $[5,6]$ ). The protein centric approach relies on the ability to purify a particular RBP or a complex thereof, followed by identification of the bound RNAs with DNA microarrays or high-throughput sequencing. Thereby, RBPs are isolated from cellular extracts with specific antibodies that selectively recognize an epitope of a constituent protein or via affinity purification of epitope tagged proteins. Whereas RBP immunoprecipitation followed by microarrays or sequencing (RIPChip/RIP-seq) involves the purification of native RNA-protein complexes under physiological conditions, the more elaborate crosslinking immunoprecipitation (CLIP) or photoactivatable ribonucleoside-enhanced crosslinking and immunoprecipitation (PARCLIP) procedures include the crosslinking of RNA-protein interactions in vivo upon exposure of cell to ultraviolet (UV)-light, followed by purification of the RBP and mild RNA digestion to obtain a footprint of the RBP binding site on the RNA $[5,6]$.

Conversely, the RNA centric approaches rely on the purification of RNA followed by the analysis of the associated proteins or RNAs. Recently, mRNA-protein interactome capture to profile the set of proteins bound to polyadenylated (poly[A]) RNAs across different organisms has become popular [7-14]. These studies confirmed hundreds of previously known RBPs, but likewise identified many novel RBPs, such as metabolic enzymes and others, whose function in post-transcriptional gene regulation is still enigmatic $[11,12,14,15]$. Nevertheless, the mapping of the RBP repertoire assembled on particular transcripts in vivo is still a challenge. Therefore, a particular mRNA shall be captured and bound proteins and RNAs identified with mass-spectrometry (MS)/ immunoblot analysis and sequencing, respectively. Currently, most applications to tackle this issue rely on the use of RNA-tags (=aptamers) that either bind to aminoglycosidic antibiotics such tobramycin or streptomycin [16-19] or to proteins with high affinity. Regarding the latter, most widely used are the coat protein from the R17/MS2 bacteriophage and streptavidin S1, a bacterial protein, both of them interacting with short hairpin RNA structures [20-24]. The repeats of the MS2 
or S1 binding RNA stem loop are appended to a RNA of interest and the tagged RNA complex is then purified by coupling of the respective protein to a solid support or resin [2024]. While these indirect RBP-mediated approaches are relatively versatile and represent an efficient tool for RNA localization studies [25-27], a major drawback is the indirect nature of the interaction, making it prone to artefacts resulting from the ectopic expression of the protein, cross-reactivity with untagged RNAs, interference with native RNP formation, and unspecific interactions. Importantly, it requires engineering of the RNA under investigation and thus cannot be applied to recover native mRNAs. Nevertheless, aptamer based approaches have been implemented successfully to enrich well-expressed RNP complexes, such as the spliceosome or ribosomal particles, whereas broader application for purification of mRNAs has been scarce.

Besides aptamers, antisense oligonucleotide (ASO) probes such as biotinylated 2'-Omethylated RNA oligos have been implemented early on for the direct purification of wellexpressed RNP complexes, such as the spliceosome [28] and telomerase [29]. More recently, modified RNA oligos were used to capture long ncRNAs (lncRNAs) from human cells [30]; whereas an array of long antisense DNA oligos were applied to capture Xist, a nuclear long ncRNA [31-32]. However, the recovery of endogenously expressed mRNAs with antisense oligos is still not well established.

Here we describe the tandem $\underline{\text { RNA }}$ isolation procedure (TRIP), a two-step procedure based on antisense DNA/RNA oligonucleotides to recover native mRNA-protein complexes from cells with high selectivity. TRIP is versatile as it was tested on particular mRNAs expressed in yeast, nematodes, and human cells, enabling further analysis of in vivo formed mRNA-protein complexes with biochemical means. In the following, we provide a detailed description of TRIP, whereby focus is given on the recovery of a particular mRNAs from poly(A) RNA with biotinylated 2'-methoxy RNA oligonucleotides.

\section{Description of the tandem RNA isolation procedure (TRIP)}

TRIP is based on the specific hybridization of nucleotide sequences in the target RNA with modified antisense DNA/RNA oligonucleotides (Fig. 1). The first step involves the enrichment of poly(A) RNAs from a cell lysate using oligo(dT) ${ }_{25}$ beads, followed by a second purification step that relies on the capturing of particular mRNAs from poly(A) RNAs using 3'-biotinylated 2'-O-methylated RNA ASOs. The bound proteins or associated RNAs on the captured mRNA are then identified by immunoblot and reverse transcription (RT)- 
PCR, respectively. In principle, the method could be extended towards the global analysis of protein and RNAs by mass-spectrometry and RNA sequencing, respectively; however, we wish to note that the set-up described herein has been designed for confirmatory purposes and was not tested for global discovery of bound proteins and/or associated RNAs, which may require substantial larger amounts of starting material.

The rationale to perform the sequential isolation of a particular mRNA, rather than a direct single step purification, is based on our attempts using either aminoglycoside-based RNA aptamers or ASOs to capture endogenously expressed mRNAs from yeast and $C$. elegans cellular extracts. Thereby, a major problem we encountered was related to the inefficient capture of respective mRNAs from crude cell extracts and contamination from unrelated proteins in the RNA isolates. Therefore, we reasoned that reducing the complexity of the samples upon capture of particular mRNAs by a first enrichment of poly(A) RNAs, which recovers mRNAs although poly(A) ncRNAs may also be present, could substantially improve recovery and specificity of the procedure. We also found that contamination with non-target mRNAs is less prevalent with TRIP.

To establish TRIP, we selected three different mRNAs from three different organisms: the endogenously expressed mRNA for PFK2 from the yeast Saccharomyces cerevisiae and cep-1 from the nematode Caenorhabditis elegans, as well as a reporter mRNA (pGL3-LUC-p27-3'UTR) bearing the 3'UTR sequence of human p27/CDKN1B mRNA fused to a luciferase reporter, which was transiently expressed in human HEK293 cells. We have chosen these mRNAs for investigation as selective mRNA-protein interactions have been reported previously, providing the opportunity to monitor the presence of respective proteins during the establishment of the procedure. The proteins used to validate this technique are: Pfk2 protein from yeast, which binds selectively to mRNAs coding for glycolytic enzymes, including PFK2 mRNA in a ribosome independent manner [11]; C. elegans GLD-1, a canonical RBP that binds to sequences located in the 3'UTR of cep-1 mRNA and thereby regulates translation [33]; and $\mathrm{HuR}$, which plays crucial roles in the regulation of mRNA stability and translation of $\mathrm{p} 27 / C D K N 1 B$ mRNA, coding for an important tumour suppressor protein [34].

\subsection{Design of antisense RNA oligonucleotides}

We favoured the use of modified RNA oligos as they allow for more stringent conditions during washing procedures and 2'-O-methyl groups offer great stability against general base hydrolysis and nucleases. Such RNA oligos have been previously applied to 
enrich ncRNAs from human cell extracts [30]; nevertheless, DNA oligos may also be feasible but have not been tested explicitly by us [35]. We thus designed several ASOs of $\sim 21-24$ nucleotides (nts) in length that specifically anneal with the desired target in the 3' untranslated regions (UTR) (Table 1). We also made oligos annealing in the open reading frame (ORF) but they generally performed less well and are thus not further described herein. Due to the property of the oligo to anneal with complementary single strands, ASOs were preferentially designed to anneal in RNA loops instead of extended double-stranded RNA regions and stems as predicted in silico with RNA secondary structure predictions programmes (http://rna.tbi.univie.ac.at/cgi-bin/RNAfold.cgi [36]. Additionally, the analysis with Basic Local Alignment Search Tool (BLAST, blast.ncbi.nlm.nih.gov/Blast.cgi) showed no significant alignment to other mRNA sequences that could lead to cross-hybridization. We wish to note that even a partial continuous alignment encompassing 8-10 nts could potentially lead to cross-hybridization and recovery of other mRNA (data not shown). After selection of several suitable ASOs for each mRNA target (we propose to design at least three ASO per RNA), the ASOs were synthesized bearing 2'-O-methyl groups, an analogue that offers stability against general base hydrolysis and nucleases, and a biotin moiety at the 3 ' end to facilitate capturing of ASOs with streptavidin beads.

\subsection{Testing selectivity of ASOs with total RNA}

To test the suitability of the ASOs for hybridization and isolation of respective mRNAs, biotinylated RNA oligos were coupled to streptavidin-conjugated paramagnetic beads and incubated with total RNA isolated from non-crosslinked organisms/cells. This approach excludes the presence of other cellular molecules such as RBPs that may mask the annealing site in the target mRNA. The amount of ASO that can be coupled to streptavidin beads was then determined empirically by titrating increasing amounts of ASOs to a fixed amount of streptavidin beads (Fig. 2A). We found that $\sim 200 \mathrm{pmol}$ (=2 $\mu \mathrm{M}$ solution) of ASO could be efficiently coupled to $30 \mu \mathrm{l}$ of streptavidin magnetic beads (M280, Invitrogen, 11205D) within $10 \mathrm{~min}$ at room temperature.

The ASO-coupled beads were further blocked with buffer containing an excess of $E$. coli derived tRNA (Roche, 10109550001) to saturate the matrix and to avoid unspecific binding of RNA. After several washes, total RNA was added to the ASO-coupled streptavidin beads and incubated for $5 \mathrm{~min}$ at $70{ }^{\circ} \mathrm{C}$ and cooled down slowly to room temperature to enable hybridization between the ASOs and the target mRNA. After washing, 
the RNA was eluted from the beads with low salt buffer (10 mM Tris-HCl, $\mathrm{pH} 7.5)$ and recovered mRNAs identified with RT-PCR. In the frame of these experiments, we realized that washing of beads at lower temperatures $\left(20-40{ }^{\circ} \mathrm{C}\right)$ could lead to the recovery of unwanted non-target mRNAs, as exemplified with cep-1 mRNA (target) and the non-target pgk-1 control mRNA (Fig. 2B). As expected, increasing the washing temperature to 50 - 55 ${ }^{\circ} \mathrm{C}$ significantly reduced contamination of the $p g k-1$ non-target mRNA in the eluate but only marginally diminished the recovery of target cep-1 mRNAs (Fig. 2B). Furthermore, we observed a progressive decrease in the capturing of target mRNAs at temperatures above 55 ${ }^{\circ} \mathrm{C}$. We thus affirm a critical role for washing at elevated temperatures, which is nevertheless below the calculated melting-temperatures for ASOs $\left(\sim 65^{\circ} \mathrm{C}\right)$.

We also tested the effect of different salt concentrations in the wash buffer at $55{ }^{\circ} \mathrm{C}$ (Fig. 2C). As shown for the yeast PFK2 target mRNA and the non-target PFK1 mRNA, we commonly observed that lowering the concentration of salt $(\mathrm{NaCl})$ in the wash buffer reduced the capture of non-target mRNAs to below detectable levels, but also substantially reduced the amount of target mRNAs ( $\sim 15 \%$ of the input). Salt concentration at near physiological levels $(\sim 150 \mathrm{mM} \mathrm{NaCl})$ performed best: about $75 \%$ of target mRNAs could be recovered and this exceeded the recovery of non-target mRNAs by at least 5-fold.

To test the versatility of the optimized procedure, we monitored the capture of selected target mRNAs and non-target mRNAs from yeast ( $S$. cerevisiae), nematodes $(C$. elegans) and human total RNA that was isolated from non-crosslinked cells/organisms (Fig. 2D). In all cases, we found that target mRNAs could be recovered with high-selectivity above non-target mRNAs with the finally selected ASOs (Table 1). Furthermore, neither mRNAs could be detected on beads in the absence of ASOs, demonstrating appropriate blocking procedures that avoid unspecific binding of RNAs to beads. We wish to note, that these experiments were useful for the evaluation of different ASOs designed for the same target mRNAs, as different oligos showed great variation in the recovery of mRNA targets (up to 5fold) and contamination with unrelated mRNAs (see Troubleshooting).

\subsection{Applying TRIP to enrich in vivo crosslinked RNP complexes}

After testing ASOs for their suitability to recover the respective mRNA target from total RNAs, we next applied the optimized conditions to recover particular mRNAs from extracts of UV crosslinked cells (Fig. 1). The irradiation of cells with UV light (254 nm) crosslinks RNA-protein interactions in vivo, therefore allowing the application of stringent wash conditions during purification. Nonetheless, although not explicitly tested, TRIP may 
also work without UV-crosslinking of cells for purification of active mRNPs applying less stringent physiological buffers. However, this bears potential for re-arrangement of proteins and/or RNAs with the target mRNA in cell-lysates, which should be taken into consideration. To evaluate selective purification of poly(A) mRNAs during the $1^{\text {st }}$ step of the procedure, we added an excess of polyadenylic acids as competitor to each extract before mRNA isolation [11]. On the one hand, we then monitored the presence of the target mRNA targets and nontarget mRNA controls by RT-PCR across the procedure (Fig 3A). On the other hand, we examined the presence of RBPs known to interact with respective mRNA targets by immunoblot analysis (Fig. 3B).

The mRNA targets from yeast, nematodes and human cells were selectively recovered from crosslinked cells across the two steps of the procedure (Fig. 3A). During the first step, the addition of poly(A) to extracts completely abolished the recovery of mRNAs and interacting proteins with oligo $(\mathrm{dT})_{25}$ beads, showing specificity for enrichment of poly(A) mRNAs. In the second step, the respective mRNA targets were preferentially captured over non-target control mRNAs with ASOs (on average $\sim 40 \%$ of target mRNAs were recovered from the input). In yeast, $P F K 2$ mRNA was enriched in final eluates whereas the non-target mRNAs PFK1 and ACT1 were not enriched; in nematodes, cep-1 mRNA was enriched at least 3-fold compared to $p g k-1$ and $m p k-1$ unrelated control mRNAs; and in HEK293 cells, mRNAs corresponding to the transiently transfected reporter harbouring the p27 3'UTR were detected, whereas the non-target $ß$-tubulin was not. As seen in test experiments with total RNA, neither mRNAs was identified on beads in the absence of ASOs, demonstrating that appropriate blocking procedures that avoid unspecific binding of RNAs. We wish to note that the recovery of non-target mRNAs may be more prevalent in the cross-linked samples compared to test experiments with non-crosslinked total RNAs as exemplified for cep-1 mRNAs (compare Fig. 3A and Fig. 2D). The reason for these differences is not known but could be manifold e.g. the masking of the binding site by other mRBPs in crosslinked samples, or the presence of RNA structures in vivo that could partially anneal with the chosen oligonucleotides.

We also followed the presence of RBPs that were expected or known to interact with corresponding mRNA targets by immunoblot analysis (Fig. 3B). We found that endogenously tandem-affinity purification (TAP)-tagged Pfk2 was recovered with PFK2 mRNA capture with ASOs. This result corroborates our earlier finding that yeast Pfk2p interacts with PFK2 mRNAs, suggesting potential post-transcriptional mediated auto-regulatory control of its expression [11]. Similarly, GLD-1 was recovered with cep-1 mRNA in C. elegans, and HuR 
was captured with reporter mRNAs bearing the 3'UTR of p27/CDKN1B mRNA from human HEK293 cells. To ensure that observed associations were genuine we also tested for nonRBPs in samples. As expected, neither Act1p from yeast $S$. cerevisiae, nor CYT-C from $C$. elegans and human $ß$-actin were enriched with respective mRNAs (Fig. 3B). These results show that TRIP is a valuable to enrich particular mRNP complexes from different species.

\section{Detailed protocol}

\subsection{Test the binding of biotinylated ASOs to streptavidin magnetic beads}

The biotinylated ASOs (Table 1) were diluted in $100 \mu \mathrm{l}$ of binding and wash (B\&W) buffer (10 mM Tris-HCl, pH 7.5, $150 \mathrm{mM} \mathrm{NaCl,} 0.5 \mathrm{mM}$ EDTA, pH 8.0) and incubated with $30 \mu 1$ (50\% slurry) streptavidin coupled magnetic beads (M280, Invitrogen, 11205D) for 10 min at room temperature with constant shaking $(500 \mathrm{rpm})$. Samples from the supernatant were taken every $10 \mathrm{~min}$. Concentration of RNA was measured by optical density (OD) at $260 \mathrm{~nm}$ with a Nanodrop ND-2000 device.

\subsection{Capture of $m R N A s$ with ASOs from total RNA}

Total RNA from yeast $S$. cerevisiae and $C$. elegans cells was isolated according to standard procedures $[37,38]$. Total RNA from HEK293 cells was isolated with the RNA Miniprep Kit (Zymo research, R1064).

To capture selected mRNAs from purified total RNA with ASOs (Fig. 2), $30 \mu 1$ (50\% slurry) of streptavidin coupled paramagnetic beads (M280, Invitrogen, 11205D) were added to a $1.5 \mathrm{ml}$ eppendorf tube and equilibrated for $1 \mathrm{~h}$ in $1 \mathrm{ml}$ of $\mathrm{B} \& \mathrm{~W}$ buffer supplemented with $0.1 \mathrm{mg} / \mathrm{ml}$ E. coli tRNA (Sigma, 10109550001) in a rotatory wheel. The beads were recovered with a magnet and resuspended and washed three times with $750 \mu 1 \mathrm{~B} \& \mathrm{~W}$ buffer at $25^{\circ} \mathrm{C}$. 200 pmol of respective ASO supplied in $100 \mu \mathrm{B} \& \mathrm{~W}$ was added to the equilibrated beads and incubated for $10 \mathrm{~min}$. The beads were subsequently washed three times with $\mathrm{B} \& \mathrm{~W}$ buffer to remove excess ASOs. $600 \mathrm{ng}$ of total RNA supplied in $100 \mu \mathrm{B} \& \mathrm{~W}$ buffer was added to the ASO-coupled streptavidin beads and incubated for $5 \mathrm{~min}$ at $70{ }^{\circ} \mathrm{C}$ in a thermoshaker incubator (Peqlab). The tubes were slowly cooled down to $25{ }^{\circ} \mathrm{C}$ and further kept at $25^{\circ} \mathrm{C}$ for $2 \mathrm{~h}$ with shaking $(1,000 \mathrm{rpm})$. Finally, the beads were washed three times in $\mathrm{B} \& \mathrm{~W}$ buffer at $55^{\circ} \mathrm{C}$ and RNA eluted in $20 \mu \mathrm{l}$ of $10 \mathrm{mM}$ Tris- $\mathrm{HCl}, \mathrm{pH} 7.5$ at $90{ }^{\circ} \mathrm{C}$ for 10 min. Specific mRNA were identified by RT-PCR with primers listed in Table 1 (see below for details). 


\subsection{First step: $U V$ crosslinking of cells and purification of poly(A) $m R N A s$}

UV crosslinking of cells, extract preparation and the isolation of poly(A) RNAs from yeast diploid cells (strain derivate of BY4743; MATa/ $\alpha$ his $3 \Delta 1 / \mathrm{his} 3 \Delta 1$ leu2 $\Delta 0 /$ leu $2 \Delta 0$ LYS2/lys $2 \Delta 0$ met15 $\Delta 0 /$ MET15 ura3 $\Delta 0 /$ ura3 $\Delta 0$ Pfk2:TAP/Pfk2) and C. elegans (Bristol N2 synchronized at larvae stage 4) was performed as described previously [11].

HEK293 cells were grown in Dulbecco's Modified Eagle Medium (DMEM) containing high glucose and pyruvate (Sigma, \#41966) and supplemented with $100 \mathrm{U} / \mathrm{ml}$ penicillin, $100 \mu \mathrm{g} / \mathrm{ml}$ streptomycin (Sigma, P4333), and $10 \%$ FBS (Sigma, F7524). The cells were grown in $100 \times 20 \mathrm{~mm}$ standard tissue culture dishes (Falcon, 353003) in a humidified incubator at $37^{\circ} \mathrm{C}$ and $5 \% \mathrm{CO}_{2} .10^{7}$ cells $(>70 \%$ confluent) were transiently transfected with $2 \mu \mathrm{g}$ of pGL3-luciferase-p27-3'UTR plasmid (I.V., A.P.G., unpublished) with $20 \mu 1$ lipofectamine 2000 (Invitrogen, 11668027) and placed in an incubator at $37{ }^{\circ} \mathrm{C}$ for $48 \mathrm{~h}$ before harvesting. Cells were washed twice with $10 \mathrm{ml}$ of pre-warmed PBS (Sigma, 59321C) and after removal the final rinse, $6 \mathrm{ml}$ of PBS was added. For crosslinking of protein-RNA complexes in vivo, the cells were exposed to UV light $(254 \mathrm{~nm})$ at $150 \mathrm{~mJ} / \mathrm{cm}^{2}$ in a Stratalinker 1800 (Stratagene). The cells were then scraped from the plate and transferred to a $15 \mathrm{ml} \mathrm{Falcon}$ tube and centrifuged at $235 \mathrm{~g}$ for $10 \mathrm{~min}$ at $4{ }^{\circ} \mathrm{C}$. PBS was removed and $2 \mathrm{ml}$ of lysis buffer (100 mM Tris-HCl, pH 7.5, $500 \mathrm{mM} \mathrm{LiCl,} 10 \mathrm{mM}$ EDTA, 1\% Triton X-100, 5 mM DTT, $20 \mathrm{U} \mathrm{ml}^{-1}$ DNase I (Promega, M6101), $100 \mathrm{U} \mathrm{ml}^{-1}$ RNasin (Promega, N2611), complete EDTA-free protease-inhibitor cocktail (Roche, 11836170001) was added to the pellet [11]. The cell lysate was then subjected to three rounds of sonication (Soniprep150, MSE) consisting of $20 \mathrm{~s}$ bursts at 10 amplitude microns and $30 \mathrm{sec}$ cooling periods on ice between bursts to complete lysis and to fragment DNA. The lysate was finally centrifuged at $15,000 \mathrm{~g}$ for $10 \mathrm{~min}$ at $4{ }^{\circ} \mathrm{C}$, and the supernatant (= extract) collected for poly(A) mRNA isolation. Protein concentration of the extract was determined with the Bradford assay (BioRad, 5000205) with BSA as a reference standard. Poly(A) RNAs were isolated from 4 $\mathrm{mg}$ of HEK293 cell extracts upon incubation with $1 \mathrm{mg}$ of oligo(dT) ${ }_{25}$ Dynabeads (Life Technologies, 61011) in lysis buffer according to our published yeast protocol [11].

3.4 Second step: Capturing of specific mRNAs with 3'-biotinylated 2'-methoxy modified antisense RNAs

$\sim 35 \mu \mathrm{g}$ (protein) of the eluate from the first step ( 15 $\mu \mathrm{g}$ poly(A) RNA) was supplied in $100 \mu \mathrm{l} \mathrm{B} \& \mathrm{~W}$ buffer and transferred to a LoBind $1.5 \mathrm{ml}$ eppendorf tube (Eppendorf, 
022431081). $200 \mathrm{pmol}$ of the corresponding ASO was added, mixed and incubated at $70{ }^{\circ} \mathrm{C}$ for $5 \mathrm{~min}$ in a thermoblock. Subsequently, the block was removed from the device and placed at room temperature $\left(\sim 25{ }^{\circ} \mathrm{C}\right)$ to cool down slowly. $30 \mu 1$ (50\% slurry) of blocked streptavidin magnetic beads (see above) were added, and the mixture was incubated for 30 min at $25{ }^{\circ} \mathrm{C}$ with constant shaking at $1,000 \mathrm{rpm}$. The beads were retrieved using a magnet and the supernatant was collected for reference. The beads were then washed three times with $750 \mu \mathrm{l} B \mathrm{~W}$ buffer at $55^{\circ} \mathrm{C}$. Finally, $50 \mu \mathrm{l}$ of $\mathrm{B} \& \mathrm{~W}$ buffer was added and the sample split: $20 \mu \mathrm{l}(2 / 5)$ for RNA and $30 \mu \mathrm{l}(3 / 5)$ for protein analysis. To recover the RNA, the B\&W buffer was removed and beads were resuspended in $20 \mu 110 \mathrm{mM}$ Tris- $\mathrm{HCl}, \mathrm{pH} 7.5$ and incubated at $90{ }^{\circ} \mathrm{C}$ for $10 \mathrm{~min}$ to elute RNA from beads. For protein analysis, the B\&W buffer was removed and beads were resuspended in $20 \mu \mathrm{l}$ Laemmli buffer $(12.5 \mathrm{mM}$ Tris-HCl, pH $6.8,4 \%$ sucrose, $1.4 \%$ SDS, $1 \% \quad \beta$-mercaptoethanol, $0.004 \%$ bromophenol blue) and denatured at $95^{\circ} \mathrm{C}$ for $5 \mathrm{~min}$.

\subsection{RNA analysis}

To perform RT, $9.4 \mu \mathrm{l}$ of the eluates from the $1^{\text {st }}$ and $2^{\text {nd }}$ step of TRIP or $500 \mathrm{ng}$ of total RNA isolated from extracts (input) were combined with $2.5 \mu \mathrm{M}$ of oligo $(\mathrm{dT})_{18}$ and 30 $\mu \mathrm{M}$ of random hexamer primers and additional compounds of the Transcriptor High Fidelity cDNA Synthesis Kit in a final volume of $20 \mu \mathrm{l}$ according to the manufactures' instructions (Roche, 05091284001). PCR was further performed with $1 \mu \mathrm{l}$ of complementary DNA (cDNA) for $10 \mathrm{~min}$ at $95{ }^{\circ} \mathrm{C}, 28-35$ cycles at $95^{\circ} \mathrm{C}$ for $30 \mathrm{sec}, 59{ }^{\circ} \mathrm{C}$ for $30 \mathrm{sec}, 72^{\circ} \mathrm{C}$ for 30 sec, and $8 \mathrm{~min}$ at $72{ }^{\circ} \mathrm{C}$ with the primers listed in Table 1 . PCR products were resolved on a $2 \%$ agarose gel and visualised with peqGreen DNA/RNA dye (Peqlab, 37-5000). Gels were recorded with a FluorChem (Alpha Innotech) and quantified with Image J software.

\subsection{Immunoblot analysis}

Proteins were resolved on 4-15\% SDS polyacrylamide gels and transferred to polyvinylidene difluoride (PVDF) membranes (Thermo Scientific Pierce, 88518). Membranes were blocked in PBS with 0.1\% Tween-20 and 5\% low fat milk, probed with the indicated antibodies and horseradish peroxidase (HRP)-coupled secondary antibodies, and developed with the Immobilon Western Chemiluminescent HRP Substrate (Millipore, WBKLS0500). The following antibodies were used: mouse anti-Act1 (1:2,500; MP Biomedicals, 0869100) for detection of yeast Act1p, mouse anti-actin (1:2,000; Sigma, A1978) for detection of human actin, mouse anti-GLD-1 (1:50, [39]), mouse anti-HuR 
(1:500; Santa Cruz, sc-5261), mouse anti-CYC-1 (1:1,000; Invitrogen, 456100), peroxidase anti-peroxidase soluble complex (1:5,000; Sigma, P1291) to detect Pfk2:TAP, and HRPconjugated sheep anti-mouse IgG (1:5,000; Amersham, NXA931).

\section{Troubleshooting}

4.1 Inefficient recovery of target mRNAs with ASOs from total RNA and/or cross-linked samples

We recommend testing several ASOs (at least three) that anneal in different regions of the transcript. In our case, we designed 2-3 ASOs of the same length that anneal in the 3'UTR or in the coding sequence of the respective mRNA target, and we noticed that each ASO exhibited different efficiency for recovery of the corresponding target. At the end, we found that ASOs annealing in the 3'UTRs performed better compared to the ones designed to coding sequences, maybe due to increased accessibility of binding sites in UTRs. We also observed repeatedly that the combination of several ASOs could lead to increased contamination from highly expressed non-target mRNAs and could decrease pull-down efficiency of the mRNA target.

It is also essential to control the integrity of the RNA in crosslinked samples as RNA degradation could lead to substantial loss of the recovery efficiency. For instance, care should be taken with UV-crosslinking as overexposure may lead to RNA degradation. Therefore, an analytical sample of the cell-lysate should be taken and RNA visualized on an agarose gel to control the integrity of total RNA. We also recommend treating the equipment with RNase decontamination solution (RNaseZap®), and the use of RNase-free consumables (tips, tubes).

Finally, we noticed the order of the addition of the components (sample-ASO-beads) was crucial for efficient recovery of RNAs from the cross-linked samples. In our hands, the incubation of the poly(A) enriched fraction with ASOs prior to the addition of streptavidin beads performed better as compared to the application of ASO-coupled beads to the sample. Possibly, the unbound oligos are kinetically favoured and have better access to sequences within structured RNA molecules.

\subsection{Contamination with unrelated RNAs or proteins in the affinity isolates}

Whereas the design of the ASOs is key for successful isolation of particular mRNAs as outlined above, other factors can also have significant impact in determining selectivity. For instance, it is essential to control the temperature of the wash buffers. We recommend to 
pre-heat the wash buffers to the desired temperature and to add them quickly to the sample, which should be kept in a thermoshaker. Furthermore, extended incubation of extracts with ASOs may lead to the increased recovery of un-related mRNAs and thus, the incubation time during annealing should be kept as short as possible. In any case, the selectivity could be further controlled by competition experiments (e.g. addition of competing oligos).

In case of a general contamination with unrelated RNAs, which is not necessarily related to the ASO, the implementation of extensive blocking procedures using BSA and/or tRNA according to the bead type specifications can reduce unspecific binding to beads. Of note, the use of lo-bind tubes is beneficial for working with low-amounts of samples, and it further reduces general background.

\section{Concluding remarks}

TRIP permits the isolation of factors bound to particular mRNAs in vivo applying biochemical means. While the method has been applied to confirm the interaction of previously known RBPs with mRNA targets, up-scaling of the procedure could enable the systematic analysis of bound proteins and/or RNAs with MS or RNA sequencing, respectively. Although the RNAs studied here were mRNAs and the RBPs associated with it, TRIP may well be applicable to study other types of poly(A) RNAs, such as cytoplasmic ncRNAs. Hence, our method complements a previously established approach to capture nuclear lncRNAs: Xist was recovered from nuclear extracts prepared from 200-800 million of cross-linked cells with an array of long (90-mer) biotinylated DNA oligonucleotides spanning the entire transcript $[31,32]$. TRIP may also be adapted for investigation of mRNA-RNA interactions, e.g. to identify the set of miRNAs that regulate a particular target transcript. Importantly, TRIP can be applied to specifically target native mRNAs without the requirement of genetic manipulation of the respective gene of interest. This is in stark contrast to currently used methodologies that use RNA aptamers to tag the respective mRNA of interest. Nevertheless, we wish to note that TRIP prerequisites the careful design and evaluation of the ASO for isolation of target mRNAs to exclude crosshybridization with related sequences (non-targets). Furthermore, RBPs/ncRNA may cover the ASO binding site in the mRNA in vivo, making it inaccessible for annealing with the ASOs. In this regard, we observed repeatedly that the combination of several ASOs could be detrimental as it can lead to increased contamination from highly expressed non-target mRNAs and decrease pull-down efficiency of the mRNA target. We thus favour the use of two sequentially arrayed ASOs 
(oligo(dT) and one specific ASO) as implemented in TRIP. In light of the rapidly increased recognition of post-transcriptional gene control, we believe that TRIP will be a formidable tool to investigate the dynamic rearrangement of RNP complexes within cells upon intracellular and environmental cues, and to understand its impact in health and disease.

\section{Acknowledgements}

We thank Dr. Jonathan Hall and Mauro Zimmermann (ETH Zurich) for the synthesis of PFK2 and cep-1 ASOs; Dr. Rafal Ciosk (Friedrich Miescher Institute for Biomedical Research, Basel) for anti-GLD-1 antibodies; Dr. Maikel Wouters for the design of p27/CDKN1B ASOs; and Drs. Nicolas Locker and Helen King (University of Surrey) for discussions. This work was supported by grants to A.P.G. from the Biotechnology and Biological Sciences Research Council (BB/K009303/1) and the Swiss National Science Foundation ('Sinergia'; CSRII3-141942).

\section{References}

[1] S. Gerstberger, M. Hafner, T. Tuschl, Nat Rev Genet, 15 (2014) 829-845.

[2] D.P. Bartel, Cell, 136 (2009) 215-233.

[3] S.F. Mitchell, R. Parker, Mol Cell, 54 (2014) 547-558.

[4] V. Iadevaia, A.P. Gerber, Biomolecules, 5 (2015) 2207-2222.

[5] C.A. McHugh, P. Russell, M. Guttman, Genome Biol, 15 (2014) 203.

[6] A.M.G. Matia-González, A.P., in: A. Sesma, von der Haar, Tobias (Ed.) Fungal RNA Biology, Springer2014, pp. 347-370.

[7] A. Castello, B. Fischer, K. Eichelbaum, R. Horos, B.M. Beckmann, C. Strein, N.E. Davey, D.T. Humphreys, T. Preiss, L.M. Steinmetz, J. Krijgsveld, M.W. Hentze, Cell, 149 (2012) 1393-1406.

[8] A.G. Baltz, M. Munschauer, B. Schwanhausser, A. Vasile, Y. Murakawa, M. Schueler, N. Youngs, D. Penfold-Brown, K. Drew, M. Milek, E. Wyler, R. Bonneau, M. Selbach, C. Dieterich, M. Landthaler, Mol Cell, 46 (2012) 674-690.

[9] S.C. Kwon, H. Yi, K. Eichelbaum, S. Fohr, B. Fischer, K.T. You, A. Castello, J. Krijgsveld, M.W. Hentze, V.N. Kim, Nat Struct Mol Biol, 20 (2013) 1122-1130.

[10] S.F. Mitchell, S. Jain, M. She, R. Parker, Nat Struct Mol Biol, 20 (2013) 127-133.

[11] A.M. Matia-Gonzalez, E.E. Laing, A.P. Gerber, Nat Struct Mol Biol, 22 (2015) 10271033.

[12] B.M. Beckmann, R. Horos, B. Fischer, A. Castello, K. Eichelbaum, A.M. Alleaume, T. Schwarzl, T. Curk, S. Foehr, W. Huber, J. Krijgsveld, M.W. Hentze, Nat Commun, 6 (2015) 10127.

[13] H.H. Wessels, K. Imami, A.G. Baltz, M. Kolinski, A. Beldovskaya, M. Selbach, S. Small, U. Ohler, M. Landthaler, Genome Res, 26 (2016) 1000-1009.

[14] Y. Liao, A. Castello, B. Fischer, S. Leicht, S. Foehr, C.K. Frese, C. Ragan, S. Kurscheid, E. Pagler, H. Yang, J. Krijgsveld, M.W. Hentze, T. Preiss, Cell reports, 16 (2016) 1456-1469. [15] A. Castello, M.W. Hentze, T. Preiss, Trends Endocrinol Metab, 26 (2015) 746-757. 
[16] K. Hamasaki, J. Killian, J. Cho, R.R. Rando, Biochemistry, 37 (1998) 656-663.

[17] M. Bachler, R. Schroeder, U. von Ahsen, RNA, 5 (1999) 1509-1516.

[18] P. Vazquez-Pianzola, H. Urlaub, R. Rivera-Pomar, Proteomics, 5 (2005) 1645-1655.

[19] K. Hartmuth, H.P. Vornlocher, R. Luhrmann, Methods Mol Biol, 257 (2004) 47-64.

[20] D.L. Beach, J.D. Keene, Methods Mol Biol, 419 (2008) 69-91.

[21] B. Slobodin, J.E. Gerst, RNA, 16 (2010) 2277-2290.

[22] B. Slobodin, J.E. Gerst, Methods Mol Biol, 714 (2011) 387-406.

[23] J.H. Yoon, M. Gorospe, Methods Mol Biol, 1421 (2016) 15-22.

[24] K. Leppek, G. Stoecklin, Nucleic Acids Res, 42 (2014) e13.

[25] E. Bertrand, P. Chartrand, M. Schaefer, S.M. Shenoy, R.H. Singer, R.M. Long, Mol Cell, 2 (1998) 437-445.

[26] D.L. Beach, E.D. Salmon, K. Bloom, Curr Biol, 9 (1999) 569-578.

[27] L. Haim, G. Zipor, S. Aronov, J.E. Gerst, Nat Methods, 4 (2007) 409-412.

[28] B.J. Blencowe, B.S. Sproat, U. Ryder, S. Barabino, A.I. Lamond, Cell, 59 (1989) 531 539.

[29] J. Lingner, T.R. Cech, Proc Natl Acad Sci U S A, 93 (1996) 10712-10717.

[30] J. Imig, A. Brunschweiger, A. Brummer, B. Guennewig, N. Mittal, S. Kishore, P. Tsikrika, A.P. Gerber, M. Zavolan, J. Hall, Nat Chem Biol, 11 (2015) 107-114.

[31] C. Chu, Q.C. Zhang, S.T. da Rocha, R.A. Flynn, M. Bharadwaj, J.M. Calabrese, T. Magnuson, E. Heard, H.Y. Chang, Cell, 161 (2015) 404-416.

[32] C.A. McHugh, C.K. Chen, A. Chow, C.F. Surka, C. Tran, P. McDonel, A. PandyaJones, M. Blanco, C. Burghard, A. Moradian, M.J. Sweredoski, A.A. Shishkin, J. Su, E.S. Lander, S. Hess, K. Plath, M. Guttman, Nature, 521 (2015) 232-236.

[33] B. Schumacher, M. Hanazawa, M.H. Lee, S. Nayak, K. Volkmann, E.R. Hofmann, M. Hengartner, T. Schedl, A. Gartner, Cell, 120 (2005) 357-368.

[34] G. Ziegeler, J. Ming, J.C. Koseki, S. Sevinc, T. Chen, S. Ergun, X. Qin, B.H. Aktas, J Biol Chem, 285 (2010) 15408-15419.

[35] T. Hassan, S.G. Smith, K. Gaughan, I.K. Oglesby, S. O'Neill, N.G. McElvaney, C.M. Greene, Nucleic Acids Res, 41 (2013) e71.

[36] A.R. Gruber, R. Lorenz, S.H. Bernhart, R. Neubock, I.L. Hofacker, Nucleic Acids Res, 36 (2008) W70-74.

[37] M.A. Collart, S. Oliviero, Curr Protoc Mol Biol, Chapter 13 (2001) Unit13 12.

[38] R. Burdine, M. Stern, Worm Breeder's Gazette1996, pp. 1.

[39] C. Scheckel, D. Gaidatzis, J.E. Wright, R. Ciosk, PLoS Genet, 8 (2012) e1002742. 


\section{Figure legends}

Fig. 1. Schematic view of TRIP. RNP complexes are crosslinked in vivo by UV-irradiation. In a first step, poly(A) RNA-protein complexes are captured with oligo $(\mathrm{dT})_{25}$ beads under stringent washing conditions. In a second step, the target mRNP complex is specifically pulled out with biotinylated anti-sense RNA oligonucleotides and streptavidin beads. The isolated mRNPs are analysed by RT-PCR and immunoblot/mass-spectrometry (MS) to identify RNAs and proteins, respectively.

Fig. 2. Testing the capture of specific mRNAs from total RNA isolated from non-crosslinked cells/organisms with antisense 3'-biotinylated 2'-methoxy RNA capture probes. (A) Time course representing the fraction of unbound ASO upon incubation with $30 \mu \mathrm{l}$ of streptavidin beads. Different lines represent different starting amounts of ASOs added to the beads. (B) Fraction of cep- 1 ASO bound mRNAs at different wash temperatures. Pgk-1 is a non-target (control) mRNA. (C) Fraction of PFK2 ASO bound yeast mRNA at different salt $(\mathrm{NaCl})$ concentration in the wash buffer. PFK1 is a non-target (control) mRNA. (D) Agarose gel showing products from RT-PCR reactions for detection of mRNAs (right) captured with indicated ASOs from yeast S. cerevisiae, C. elegans, and human HEK293 (H. sapiens) total RNA. Input, total RNA; Sn, supernatant after incubation with ASOs; E, eluates from beads; Ctrl, control experiment performed in parallel without addition of ASOs.

Fig. 3. Isolation of specific mRNA-protein complexes from cell extracts of UV cross-linked organisms or human cells with TRIP (A) Agarose gel showing products from RT-PCR reactions for detection of mRNAs (right) captured with oligo(dT) and indicated ASOs from $S$. cerevisiae, C. elegans and human (H. sapiens) cell extracts. Input, total RNA isolated from crosslinked cells/organisms; Ctrl, control experiment (Ctrl) without addition of ASO. Poly(A), addition of competitor poly(A) to evaluate the specificity of mRNA isolation. (B) Immunoblot analysis for detection of mRNA-bound proteins with specific antibodies (right). The following amounts were loaded on the gel: $0.1 \%, 2.5 \%$ and $1 \%$ of the yeast, nematode and human extract (input); 10\%, 10\% and 5\% of the yeast, nematode and human oligo(dT) isolates; and $66 \%$ of the ASO and Ctrl eluates. Markers with molecular weights (MW) are indicated in kilodaltons $(\mathrm{kDa})$. 
Table 1: Oligonucleotide sequences.

\begin{tabular}{|c|c|c|c|}
\hline Primer name & Sequence (5'-3') & Target & Length \\
\hline Pfk2_Fwd & GTGTTAAGGGTTCACATGTCG & PFK2 S. cerevisiae & $133 \mathrm{bp}$ \\
\hline Pfk2_Rev & CTTCCAACCAAATGGTCAGC & PFK2 S. cerevisiae & $133 \mathrm{bp}$ \\
\hline Pfk1_Fwd & GGTGATTCTCCAGGTATGAATG & PFK1 S. cerevisiae & $97 \mathrm{bp}$ \\
\hline Pfk1_Rev & CTTCGTAACCTTCGTAAACAGC & PFK1 S. cerevisiae & $97 \mathrm{bp}$ \\
\hline Act1_Fwd & GTCTGGATTGGTGGTTCTATC & ACT1 S. cerevisiae & $85 \mathrm{bp}$ \\
\hline Act1_Rev & GGACCACTTTCGTCGTATTC & ACT1 S. cerevisiae & $85 \mathrm{bp}$ \\
\hline Cep1_Fwd & CGATGAAGAGAAGTCGCTGT & cep-1 C. elegans & $110 \mathrm{bp}$ \\
\hline Cep1_Rev & ATCTGGGAACTTTTGCTTCG & cep-1 C. elegans & $110 \mathrm{bp}$ \\
\hline Pgk1_Fwd & GCGATATTTATGTCAATGATGCTTTC & pgk-1 C. elegans & $74 \mathrm{bp}$ \\
\hline Pgk1_Rev & TGAGTGCTCGACTCCAACCA & pgk-1 C. elegans & $74 \mathrm{bp}$ \\
\hline Mpk1_Fwd & TGCTCAGTAATCGGCCATTG & mpk-1 C. elegans & $74 \mathrm{bp}$ \\
\hline Mpk1_Rev & TCCAACAACTGCCAAAATCAAA & mpk-1 C. elegans & $74 \mathrm{bp}$ \\
\hline p27_Fwd & $\begin{array}{l}\text { TTTAAAAATACATATCGCTGA } \\
\text { CTTCATGG }\end{array}$ & $\begin{array}{l}\text { p27 } \\
\text { H. sapiens }\end{array}$ & $212 b p$ \\
\hline 27 Day & CAAAGTTTATGTGCTACATAA & $p 27$ & \\
\hline p27_Rev & AAGGTAAAAA & H. sapiens & $212 \mathrm{bp}$ \\
\hline Luc_Fwd & AATGGCTCATATCGCTCCTGGAT & Luciferase P. pyralis & $117 \mathrm{bp}$ \\
\hline Luc_Rev & TGGACGATGGCCTTGATCTTGTCT & Luciferase P. pyralis & $117 \mathrm{bp}$ \\
\hline$\beta$-TUB_Fwd & CTGAACCACCTTGTCTCAGC & $\beta$-TUB H. sapiens & $136 \mathrm{bp}$ \\
\hline$\beta$-TUB_Rev & AGCCAGGCATAAAGAAATGG & $\beta$-TUB $H$. sapiens & $136 \mathrm{bp}$ \\
\hline$P F K 2$ ASO & GUUUCAUGGGGUAGUACUUGU & $\begin{array}{l}\text { 3' UTR PFK2 } \\
\text { S. cerevisiae }\end{array}$ & - \\
\hline cep-1 ASO & GUGAGAAAUGCGGUGCUUUGAAA & $\begin{array}{l}\text { 3' UTR cep-1 } \\
\text { C. elegans }\end{array}$ & - \\
\hline p27 ASO & UCAUACCCCGCUCCACGUCAGUU & $\begin{array}{l}\text { 3' UTR p27 } \\
\text { H. sapiens }\end{array}$ & - \\
\hline
\end{tabular}


UV irradiation (254 nm)
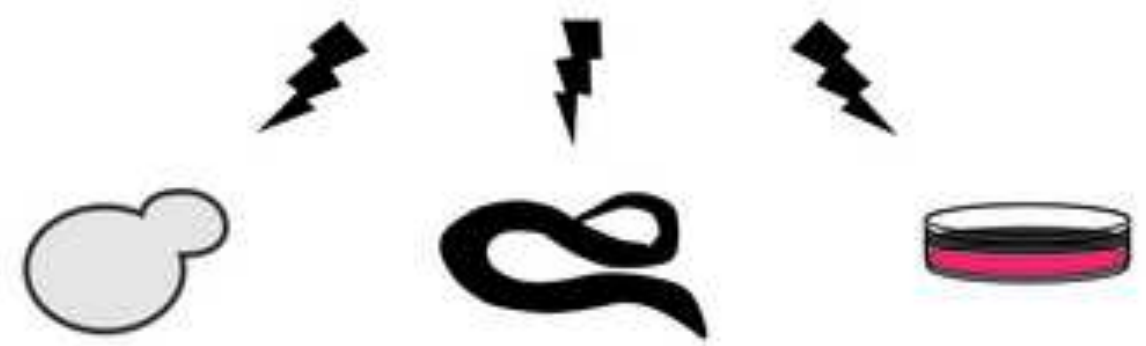

Saccharomyces cerevisiae

Caenorhabditis

HEK293 cells elegans (Homo sapiens)
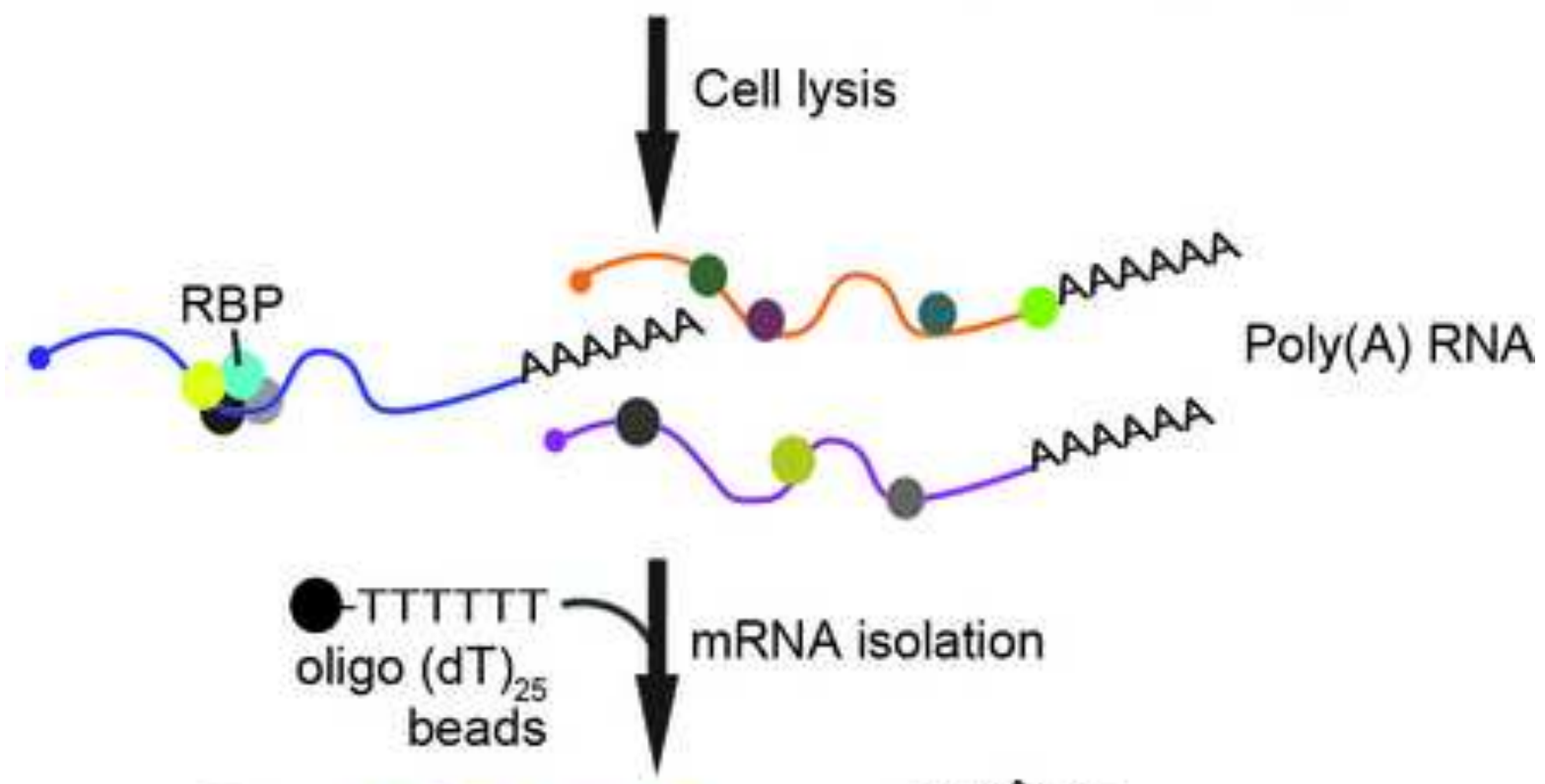

(1) $\sim$ TAAAATO Biotin Oligo annealing to mRNA
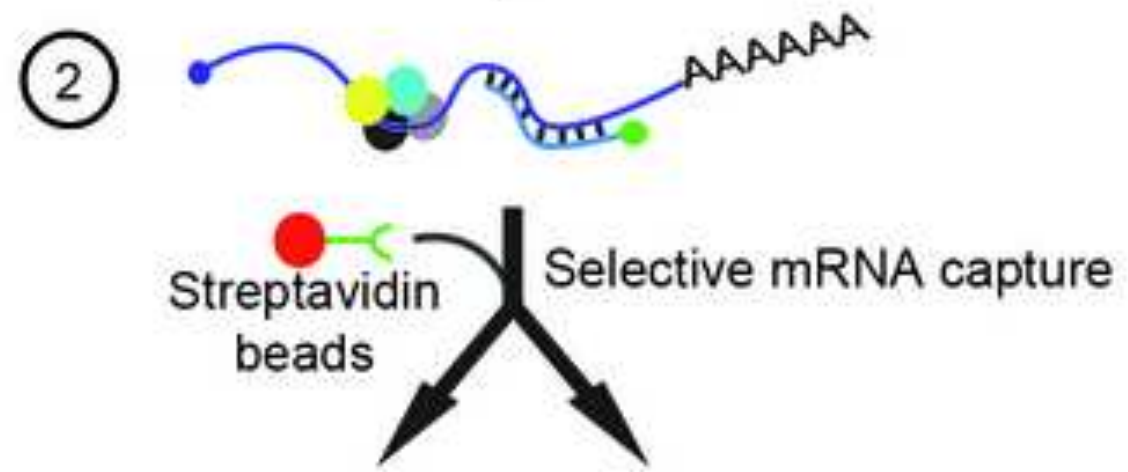

RNA analysis

Protein analysis

RT-PCR

Immunoblot

Mass-spectrometry 
A

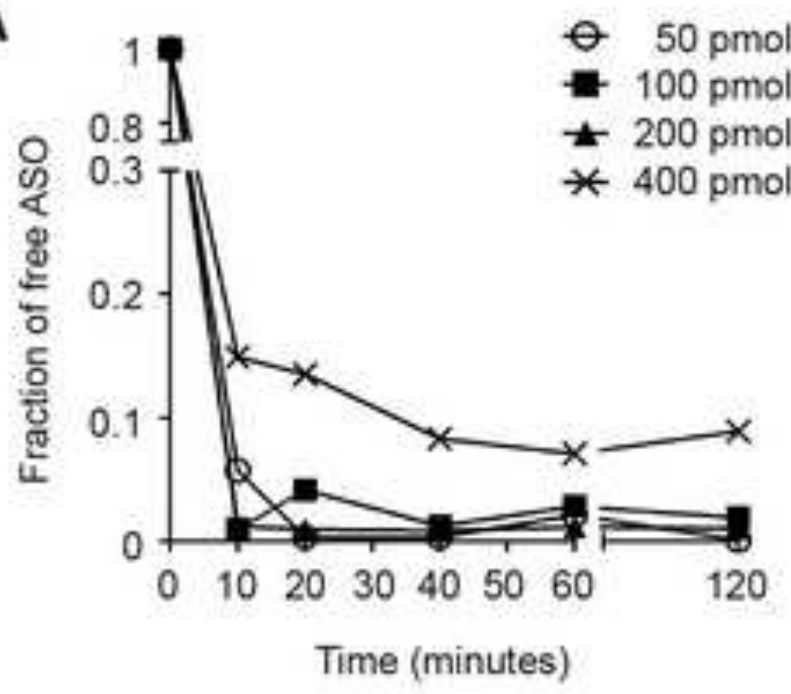

B

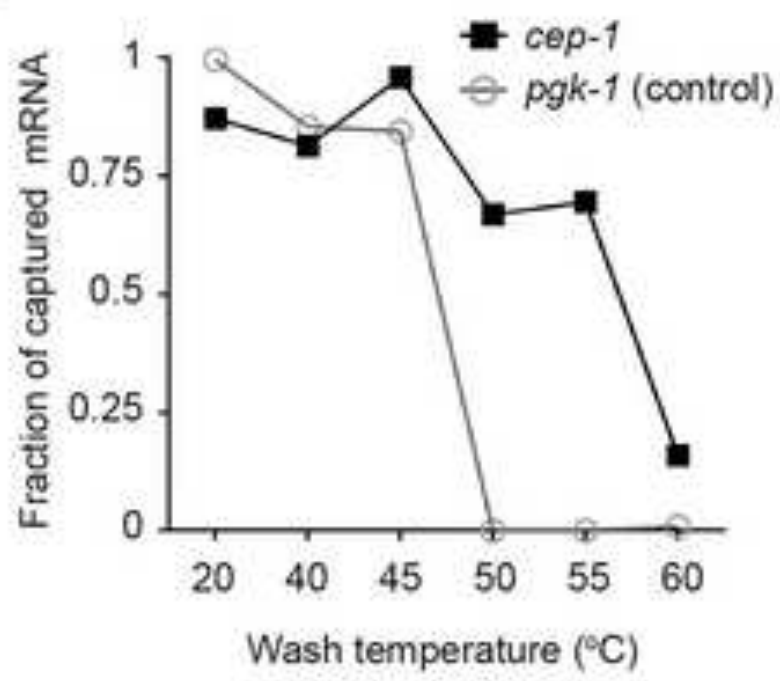

C

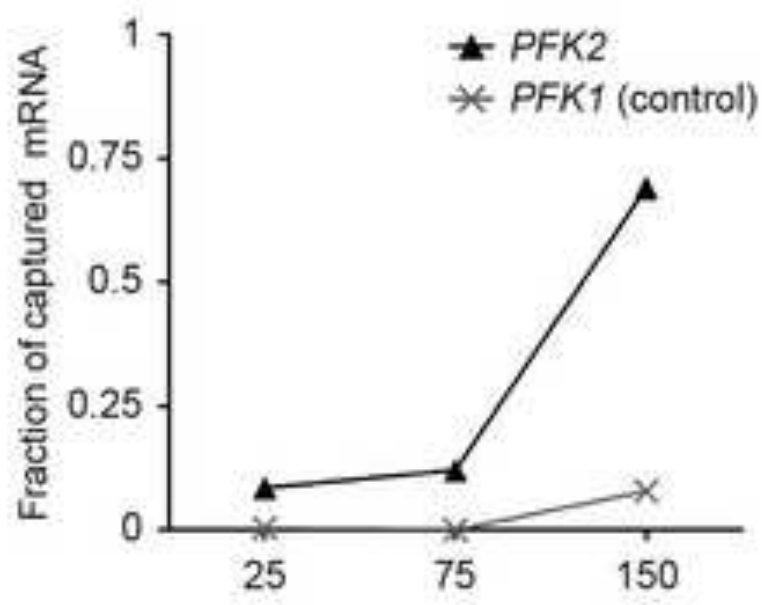

Salt concentration (mM)
S. cerevisiae

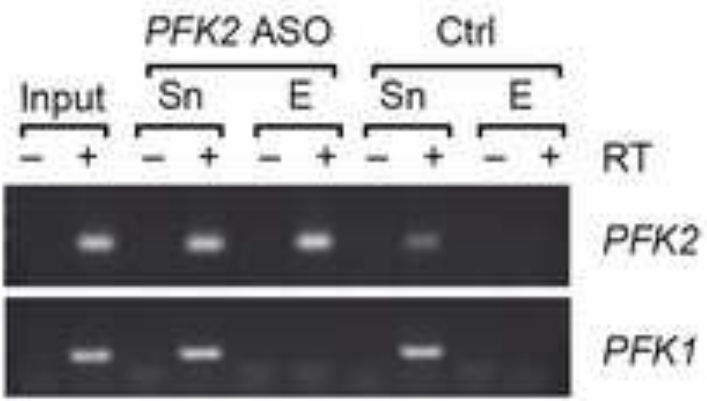

C. elegans

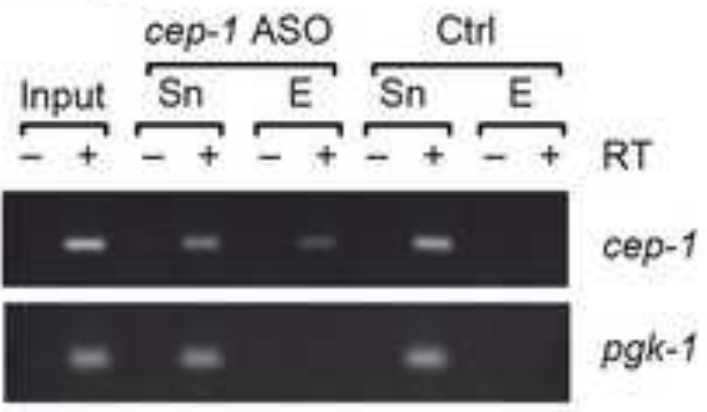

H. sapiens

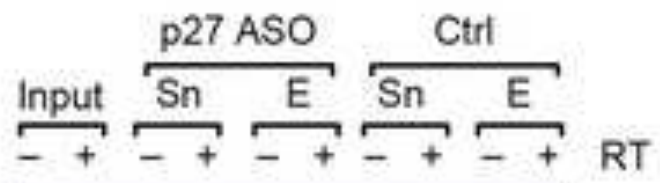

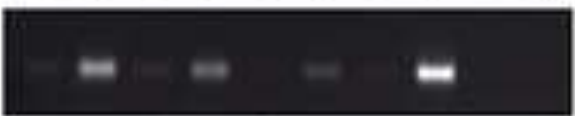
p27/CDKN1B

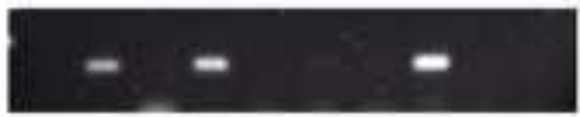

B-TUB 
A S. cerevisiae

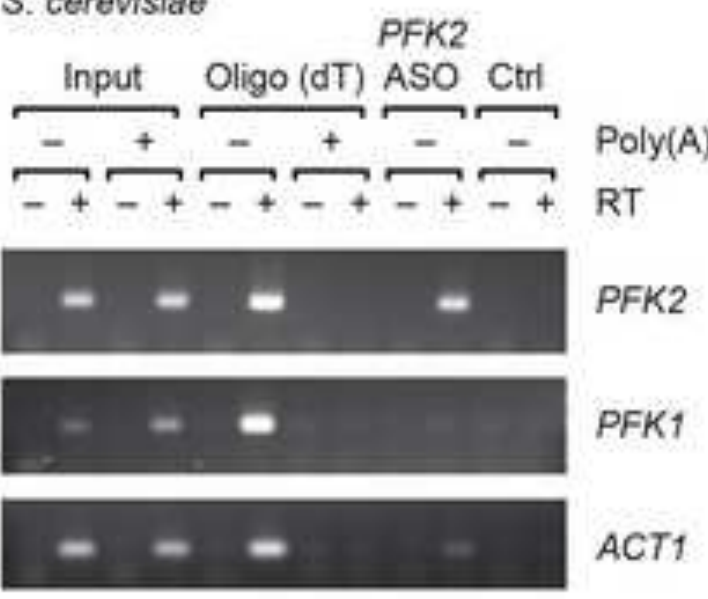

C. elegans

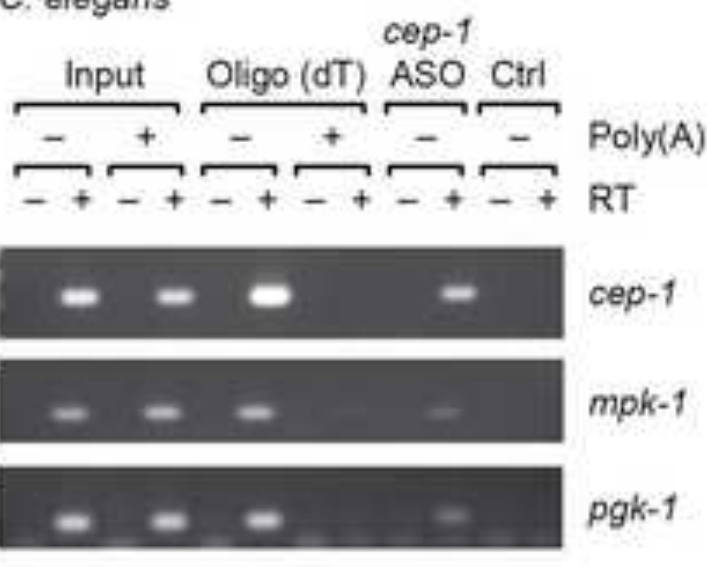

H. sapiens

p27.

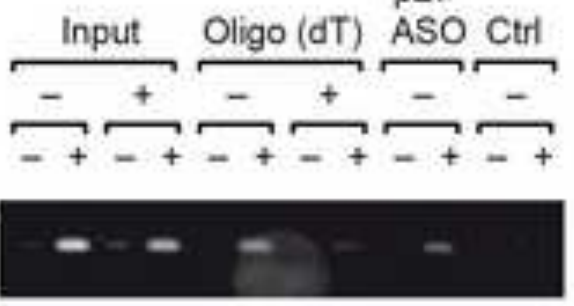

Poly(A)

RT

LUC

-p27/CDKN1B

primer dimer

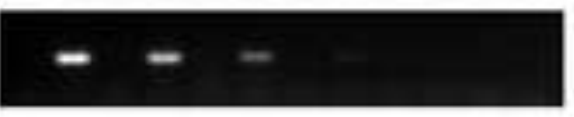

B-TUB
B S. cerevisiae

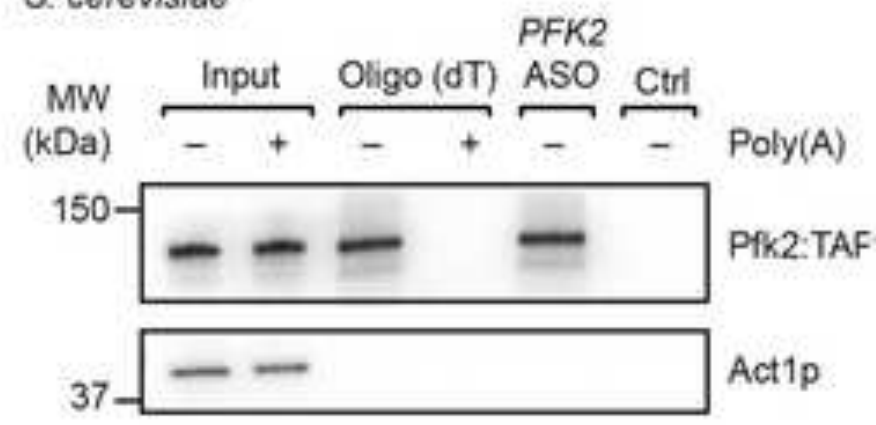

C. elegans

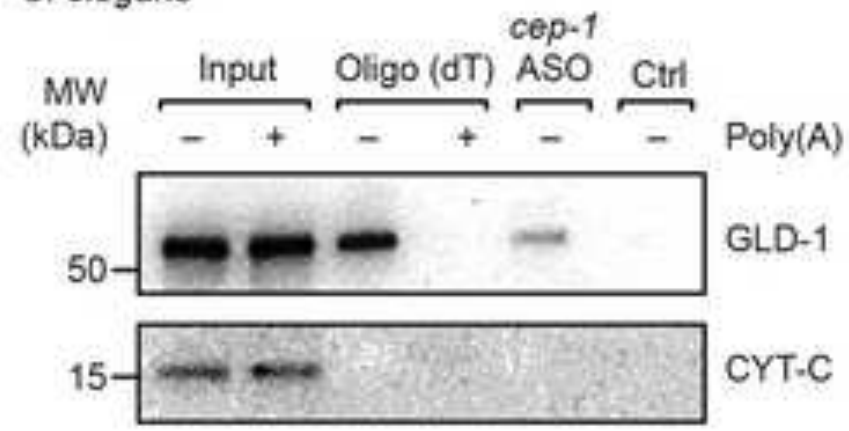

H. sapiens

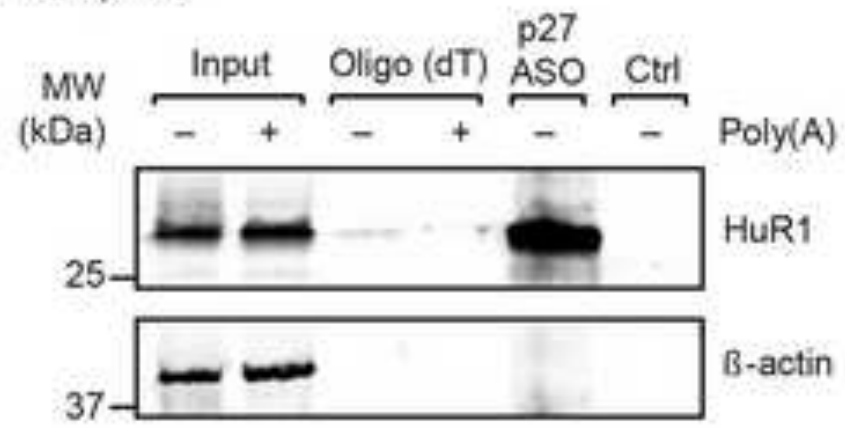

